\title{
Software livre de produção textual Com predição de palavras: UM ALIADO DO ALUNO ESPECIAL
}

\author{
TEXT PRODUCTION WITH A WORD PREDICITION FREE-WARE SOFTWARE: \\ SUPPORT FOR THE SPECIAL STUDENT
}

\author{
Monica JORDAN ${ }^{1}$ \\ Percy NOHAMA2 \\ Alceu de Souza BRITTO JÚNIOR
}

\begin{abstract}
RESUM 0: a dificuldade em comunicar-se naturalmente, seja através da fala ou da escrita, pode fazer com que uma pessoa seja impedida de demonstrar e desenvolver seus potenciais cognitivos, sociais e criativos. Essa realidade tem sido amplamente estudada, buscando cada vez mais e melhores soluções que contornem tal limitação, pela ciência multidisciplinar conhecida como Comunicação Alternativa e Aumentativa. Dentre as soluções encontradas, algumas delas fazem uso do computador como ferramenta de apoio à comunicação. A predição de palavras e simuladores de teclado para produção textual estão entre essas soluções. Este artigo vem divulgar e discutir os resultados obtidos com a aplicação de um simulador de teclado em conjunto com uma técnica de predição de palavras baseada nos grupos das classes gramaticais da língua portuguesa, o qual oferece ao usuário facilitação na produção de textos com qualidade gramatical. Dados obtidos com adolescentes com seqüelas de paralisia cerebral mostraram que o uso do aplicativo é construtivo, amplia a comunicação e pode auxiliar no processo de aprendizado da língua portuguesa. Ampliar a comunicação sem prejudicar a qualidade gramatical é uma forma de auxiliar no processo de educação e inclusão social de pessoas com deficiência, tornando-as socialmente mais participativas e melhorando a qualidade de suas vidas.
\end{abstract}

PALAVRAS-CHAVE: educação especial; software; educação inclusiva; informática educativa.

ABSTRACT: the difficulty in communicating naturally, through speech or writing, can impede a person demonstrating and developing his or her cognitive, social and creative abilities. This reality has been thoroughly studied, looking for more and better solutions to circumvent said limitation, using the multidisciplinary science known as Alternative and Augmentative Communication. Among the proposed solutions, some use computers as a tool to support communication. Word prediction and on-screen keyboard simulators used for writing are among such facilitators. This paper presents and discusses the results obtained when keyboard simulator were applied along with word prediction techniques based on grammatical class groups for the Portuguese language; this proposal facilitates writing production with good grammatical quality. Data obtained with adolescents with sequelae from cerebral palsy showed that the use of the software was positive, as it enhanced communication and was useful in the learning process for the Portuguese language. Broadening communication possibilities without prejudice to grammatical quality is a means of aiding in the educational process and social inclusion for people with special needs, so as to enhance social participation and improve quality of life.

KEYWORD S: Special Education; Inclusive Education; Computer Education.

\footnotetext{
${ }^{1}$ Mestre em Ciências, Universidade Tecnológica Federal do Paraná, CPGEI, mjordan_30@yahoo.com.br ${ }^{2}$ Doutor em Engenharia de Engenharia Elétrica, Universidade Tecnológica Federal do Paraná, CPGEI, percy@cpgei.ct.utfpr.edu.br

${ }^{3}$ Doutor em Informática, Pontifícia Universidade Católica do Paraná, PPGIA, alceu@ppgia.pucpr.br
} 


\section{INTRODUÇÃo}

O censo de 2000 apontou que cerca de 24,5 milhões de brasileiros apresentam al gum tipo de deficiência (IBGE, 2000). Com base nestemesmo censo, Neri e Soares (2004) apresentaram um estudo com dados mais detalhados, resumidos nas proporções de população para cada deficiência encontrada, as quais dizem que $11,56 \%$ das pessoas com alguma deficiência apresentam deficiência mental; 0,44\% apresentam tetraplegia, paraplegia ou hemiplegia; $22,70 \%$ possuem alguma dificuldade para caminhar ou subir escadas; $7,21 \%$ relataram grande dificuldade e 2,30\% disseram-se incapazes; 5,32\% apresentam ausência de algum membro ou de parte dele; quanto à visão, 57,16\% relataram al guma dificuldade para enxergar; $9,90 \%$ grande dificuldade e 0,60\% são incapazes de enxergar; quanto à audição, 19,05\% apresentaram alguma dificuldade para ouvir; 3,59\% apresentaram grande dificuldade e 0,68\% são incapazes.

Algumas das deficiências citadas acabam deteriorando a comunicação ou são acompanhadas da dificuldade em se comunicar. Uma em cada duzentas pessoas, no mundo, apresenta dificuldade verbal (CAPOVILLA, 1994). Essa dificuldade pode ser compensada pela comunicação escrita. No entanto, sabe-se que a afasia, alteração de linguagem adquirida por uma lesão cerebral focal, pode estar associada a outras manifestações neurológicas ou neuropsicológicas, como transtornos motores (hemiparesia ou hemiplegia) que atinge $80 \%$ dos afásicos não-fluentes e $20 \%$ dos afásicos fluentes, transtornos sensoriais, visuais, demência eal terações decomportamento (PEÑ A-CASA NOVA e PAMIES, 2005). Essas manifestações podem afetar a comunicação escrita e têm-se, então, pessoas com extrema dificuldade em se comunicar.

Com o intuito de superar as limitações impostas à pessoa que tem dificuldade na comunicação, seja escrita ou oral, faz-se necessário o emprego de sistemas alternativos de comunicação. O termo alternativo pode estar ligado a vários significados como o de proporcionar economia nos esforços envolvidos na comuni cação em termos de atenção, entusiasmo e recompensa (MORETTI, 1999). O desenvolvimento desses sistemas é orientado pela Comunicação Alternativa e Aumentativa (CAA) ou Augmentative A Iternative Communication (AAC), sigla cunhada na década de 80, nos Estados Unidos da A mérica na ocorrência da instituição de uma associação internacional chamada ISAAC (International Society for Augmentative AIternative Communication), fruto do trabalho multidisciplinar que objetiva o direito de comunicação mesmo para pessoas com grave impedimento verbal (GAVA, 1999).

Lucchini (2001) afirma que o uso de aparatos tecnológicos de Comunicação Alternativa no ensino especial não se refere à correção de algum tipo de anomalia intelectual, mas sim a oferta de um apoio que facilite a exteriorização edesenvol vimento de seus potenciais cognitivo, criativo ehumano. 
Dessa forma, a presença dos computadores nas salas de aula tem sido encarada como uma importante ferramenta de auxílio, a qual tem sustentação nas terapias que adotam as máquinas como auxiliares. Crianças tratadas com auxílio do computador motivam-se e concentram-se mais. Os elementos básicos do funcionamento mental são estimulados pelas cores e sons da máquina, o que facilita a compreensão das atividades a serem executadas. Ainda, por ser a resposta dada pela criança através do computador completamente entendida pel o terapeuta, independente de escuta, escrita, desenhos, etc., evita-se repetição de tarefas já superadas pela criança e gera-se uma maior interação com a tarefa e seu objetivo comunicativo e terapêutico (STELLA, 1999).

Mostrando-se diferente às análises iniciais sobreo uso do computador na sala de aula, Schlünzen et al. (2000) apontam que esta prática não prescinde da presença do professor no ambiente educacional, mas que amplia sua ação fazendo-o atuar como mediador entre a máquina e o aluno. A presença do educador em momento al gum é menos importante com a adoção da informática, pois cabe a ele orientar, estimular, avaliar e ensinar esta ferramenta de aprendizagem. $\mathrm{O}$ computador deve apresentar-se como um instrumento que 0 educador adiciona ao seu conjunto de métodos pedagógicos, não privando o processo de ensino-aprendizagem das relações humanas, emoção e afetividade.

Stella (1999) narra que, em sua experiência profissional, os aprendizados estimulados pela máquina ultrapassam as atividades manuais, alcançando diferentes estruturas mentais e ativando novas formas de funcionamento.

Todos os relatos citados até aqui motivaram e justificam o projeto implementado, no qual desenvolveu-se uma técnica para auxiliar a produção textual de crianças com dificuldades na comunicação. A técnica descrita neste artigo baseia-se nas regras gramaticais da língua portuguesa e, além de facilitar a produção de textos, consegue ampliar o vocabulário e despertar a curiosidade por novas palavras que são apresentadas aos seus usuários. O uso deste tipo de predição mostra melhoria na quantidade e qualidade de comunicação, como apontada no trabal ho AMPLISOFT (JORDAN; MATIAS; NOHAMA, 2006). A reutilização de textos por usuários capazes de incorporar novos textos é eficiente e facilitadora (ALM, 1997).

O aplicativo resultante baseia-se num simulador de teclado na tela do computador que apresenta, ao lado direito das letras, um campo no qual são indicadas palavras que tentam antecipar ao usuário a próxima palavra a ser digitada ou completar a que está sendo digitada.

\section{MÉtodo}

O trabal ho descreve um software desenvolvido com base nos aplicativos de programação M icrosoft V isual Basic $c^{\circledast}$, Excel $2003^{\circledR}$ e M icrosoft V isual 
$\mathrm{C}++^{\oplus}$, baseado em uma lista de textos pré-selecionados, denominada corpus (SARDINHA, 2004), e no processo estocástico conhecido por Modelos Ocultos de Markov (RABINER, 1989).

Inicialmente, ocorreu a estruturação do corpus, para o qual foram selecionados na web cem textos infantis que apresentassem o mínimo possível de erros ortográficos e gramaticais, poucas figuras de linguagem e evitassem o uso exagerado degírias. Crio-se um corpus com 40.329 pal avras, sendo 7066 diferentes. Todas as palavras do corpus foram etiquetadas com suas respectivas classes gramaticais e inflexões, com auxílio do Visual Interactive Syntax Learning (VISL).

Buscou-se formar um corpus que contivesse:

1. lendas brasileiras com personagens comuns à literatura infantil;

2. textos de cultura popular, com histórias regionais;

3. diálogos;

4. textos religiosos de linguagem infantil que fizessem referência a Deus, Jesus e Maria, personagens conhecidos por crianças de diferentes religiões;

5. fábulas;

6. nomes de animais mais citados em histórias infantis;

7. expressões populares;

8. corpos da natureza: árvore, mar, cachoeira, rio, Sol, Lua, etc.;

9. objetos do dia-a-dia doméstico;

10. cômodos da casa;

11. nomes de flores comuns, como margarida e rosa.

Com a base de textos finalizada partiu-se à determinação dos parâmetros para a análise estatística das palavras a serem preditas no aplicativo.

Os Modelos Ocultos deMarkov estruturam-senos seguintes elementos:

1. conjunto dos $\mathrm{N}$ estados $\left\{S_{j}\right\}$, caracterizado no trabalho como sendo o conjunto das 254 classes gramaticais identificadas no corpus;

2. matriz de transições $A=\left\{a_{i j}\right\}$, sendo a transição que ocorre entre as classes gramaticais do corpus;

3. comprimento da sequencia observada (número de símbolos mostrados nela), representado por T edeterminada pelo número máximo de pal avras presentes em uma mesma frase do corpus, sendo igual a 100; 
4. sequência de observações $O=\left\{o_{1}, o_{2}, \ldots, o_{T}\right\}$, representadas pelas palavras;

5. número $M$ de possíveis observações para os estados, constituindo o conjunto $V=\left\{v_{1}, v_{2}, \ldots, v_{M}\right\}$. Como o estado é representado por uma classe gramatical, as observações possíveis para cada estado são as palavras do corpus etiquetadas com a referida classe gramatical;

6. matriz de ocorrências contendo as probabilidades de saída $B=\left\{b_{j k}\right\}$, na qual os elementos bjk definem a probabilidade de ocorrência do símbolo k no estado j;

7. matriz inicial $\pi$ contendo a probabilidade de cada estado iniciar uma sequência, ou seja, das palavras que iniciam sentenças.

Desta forma, puderam-se delinear as situações em que o texto digitado poder-se-ia encontrar e, para cada condição, como a predição deve ser realizada.

As seguintes situações foram consideradas:

1. início de sentença;

2. predição após digitada uma palavra conhecida;

3. predição após uma palavra desconhecida;

4. ato de completar a palavra sendo digitada.

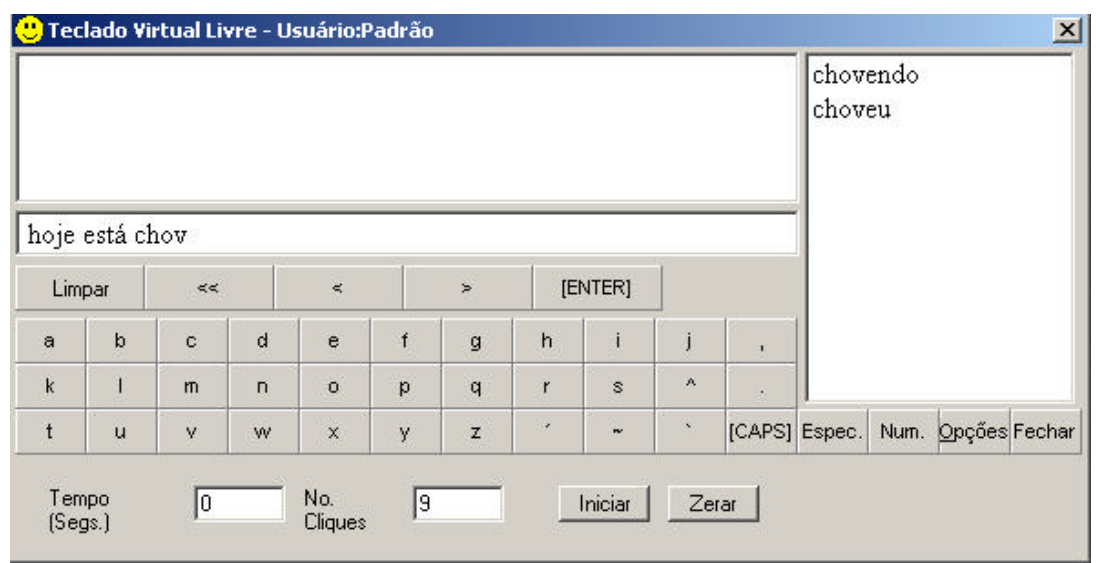

Figura 1 - Interface gráfica do simulador de teclado empregado nos testes, denominado Teclado Virtual Livre (JORDAN; MATIAS; NOHAMA, 2006). 
A cada situação o aplicativo processa o estado e apresenta as palavras mais prováveis de ocorrerem para o dado momento, num número máximo de 10 palavras. Caso a palavra desejada apareça na lista de predição, apresentada no quadro à esquerda do aplicativo (Figura 1), basta que o usuário clique sobre a mesma. Em caso negativo, segue-se a digitação e a cada letra digitada um novo processamento é realizado até que se encerre a digitação de um vocábulo, reconhecido pela seleção da tecla "espaço", ou por alguma pontuação. O processo descrito é o mesmo exigido pelo Teclado Virtual Livre do projeto AMPLISOFT, o qual se encontra disponível e detalhado em www.ler.pucpr.br/ amplisoft.

Atualmente, o aplicativo não aceita a inclusão de novas palavras devido ao seu processamento estocástico. No entanto, tem-se o intuito de continuamente ao projeto, desenvolver-seum aplicativo com inteligência artificial, que possa aceitar palavras novas. Este é um processo muito mais detalhado, pois o algoritmo terá que rotular a palavra inserida, classificando-a gramaticalmente.

Desenvolvido o algoritmo, realizaram-se testes com alunos de duas escolas, uma de ensino especial e outra de ensino fundamental.

Para verificar a aplicabilidade e vantagem do uso da técnica de predição no simulador de teclado adotado ilustrado na Figura 1, foi realizado testecom dois grupos: um grupo de 41 crianças de diferentes realidades cognitivas, segundo relato da professora de informática e da coordenadora de ensino da escola, e um grupo de 10 adolescentes alunos de uma escola de ensino especial. Tal teste consistia no uso do computador para produção do texto "A Lagarta" de Leonor Von Osterroht, extraído do sítio http:/ / www.usinadeletras.com.br/ , com auxílio do aplicativo. A pós demonstração do aplicativo na tela do computador, era solicitado ao usuário que digitasse um determinado texto, por duas vezes, sendo a primeira vez sem a predição de palavras e a segunda com. Cita-se que por se tratar de um simulador de teclado o entendimento do uso por pessoas familiarizadas aos computadores é simples, dispensando maiores explicações. Já os voluntários deficientes já eram conhecedores do aplicativo AMPLISOFT (JORDAN; MATIAS; NOHAMA, 2006).

O texto "A Lagarta" foi selecionado por encontrar-se em sítio reconhecido pela Unesco e apresentar em apenas 40 palavras um enredo com começo, meio e fim. O texto ao todo necessita de 236 caracteres (considerados os espaços entre palavras), para ser digitado.

Para a realização dos testes o texto fora entregue aos voluntários impresso em folha de papel branco, em fontetipo "Verdana", fonte 14. A posição do papel para leitura era livre, não havendo suportes ou apoios especiais.

Foram anotados o número de cliques e o tempo (em segundos) despendido para a execução das tarefas, possíveis de visualização na Figura 1, na base do simulador de teclado. Todos os testes foram acompanhados de uma pedagoga, fonoaudióloga ou fisioterapeuta da instituição onde se realizaram os 
testes e aprovados pelo Comitê de Ética da Pontifícia Universidade Católica do Paraná (Of. 731/ 06/ CEP-PUCPR).

A amostra foi composta por quatro grupos distintos, sendo três deles formados pelos alunos das três turmas de $4^{a}$ série da escola de ensino fundamental e o outro grupo formado por alunos alfabetizados e com habilidade cognitiva suficiente para utilizar o aplicativo e digitar um texto, da escola deensino especial, contendo cada um 11, 11, 12 e 7 alunos.

O tamanho da amostra foi determinado pelo número de crianças autorizadas pelos responsáveis a real izar os testes e que estiveram presentes nas aulas de informática em que os testes foram realizados. Foi considerado grupo de controle o conjunto de resultados obtidos sem o uso da técnica de predição.

Os parâmetros tempo e número de cliques disponibilizados pelo próprio aplicativo, durante seu uso, foram analisados comparativamente, obtendo-se um percentual de redução ou incremento dos parâmetros com e sem o uso da predição.

A análise baseou-se, também, em comparação direta dos resultados obtidos, determinando-se, em percentual, a redução ou aumento dos parâmetros envolvidos. E a comparação de valores obtidos com os encontrados na literatura referente.

foram:

Os critérios de exclusão para a determinação do grupo de voluntários

1. não ser alfabetizado;

2. estar impossibilitado de controlar o computador através do periférico mouse convencional ou dispositivos auxiliares;

3. não estar participando de aulas de informática dentro de sua respectiva instituição de ensino.

Já o grupo formado pelos voluntários da escola de ensino especial foi formado por meio dos seguintes critérios:

1. ser aluno regular da escola;

2. apresentar boa freqüência às aulas (acima de $70 \%$ );

3. apresentar dificuldade na comunicação natural (fala, gestos ou sinais);

4. ser aluno das aulas de informática oferecida pela escola, ou seja, estar adaptado ao uso do computador;

5. ser alfabetizado.

Sob decisão da pedagoga responsável da escola de ensino especial, 10 voluntários foram selecionados para a realização dos testes. Este grupo foi formado por adolescentes, todos alfabetizados, com paralisia cerebral e sem 
dificuldades visuais, segundo informações da escola onde foram realizados os testes. Destaca-se que a seleção dos candidatos e a aplicação dos testes foi realizada, na íntegra, pela própria instituição de ensino, considerando os critérios e as recomendações citados anteriormente.

O nível de paralisia cerebral, bem como as limitações físicas impostas ao uso do computador não foram avaliados, pois seriam irrelevantes ao trabalho. Já as condições cognitivas e o comprometimento motor dos voluntários foram apontados pela pedagoga responsável como equivalente entre eles.

Os alunos da escola de ensino especial utilizaram, conforme suas necessidades, periféricos alternativos, como mouse de cabeça e acionadores (JORDAN; MATIAS; NOHAMA, 2006), todos pertencentes à escola e usuais aos voluntários.

\section{Resultados}

Os testes de aplicabilidade, realizados por 41 voluntários indicaram os resultados das tarefas de digitação sem e com o uso da predição de palavras, apresentados nas Figuras 2 a 5, sendo as três primeiras obtidas na escola de ensino fundamental e a última na escola de ensino especial.

Com base nos dados obtidos com o primeiro grupo, foram gerados dois gráficos para análise comparativa dos parâmetros tomados nos testes, os quais são ilustrados pelas Figuras 2 e 3 . A Figura 2 ilustra um gráfico onde o eixo horizontal corresponde aos voluntários e o vertical, ao parâmetro tempo.

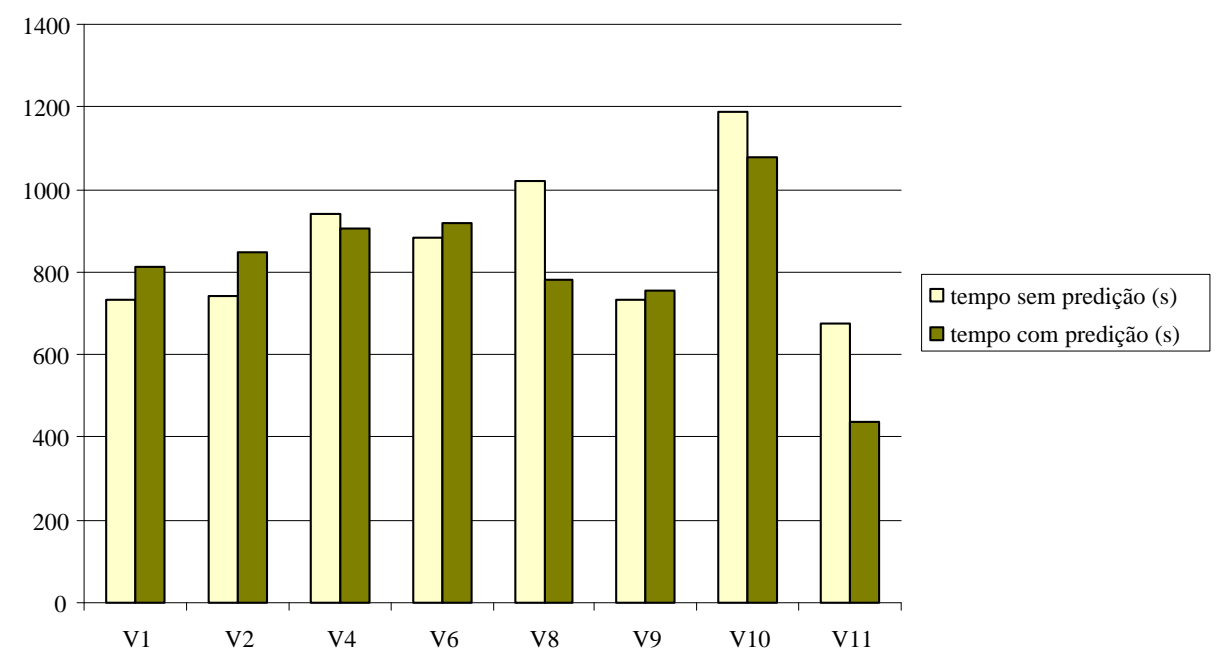

Figura 2 - Grupo 1: comparação do tempo gasto para digitação do texto com e sem predição. 
N ota-se que em todos os gráficos expostos, as siglas $V 1, \mathrm{~V} 2, \ldots$, etc., do eixo horizontal, referem-se à, respectivamente, Voluntário 1, Voluntário 2, ..., etc., e que o número de voluntários dos gráficos às vezes é diferente do tamanho da amostra; isto deve-se ao fato dequenem todos os dados obtidos foram passíveis de análise comparativa.

Para o parâmetro número de cliques, gerou-se o gráfico exposto na Figura 3, no qual o eixo vertical corresponde ao parâmetro número de cliques.

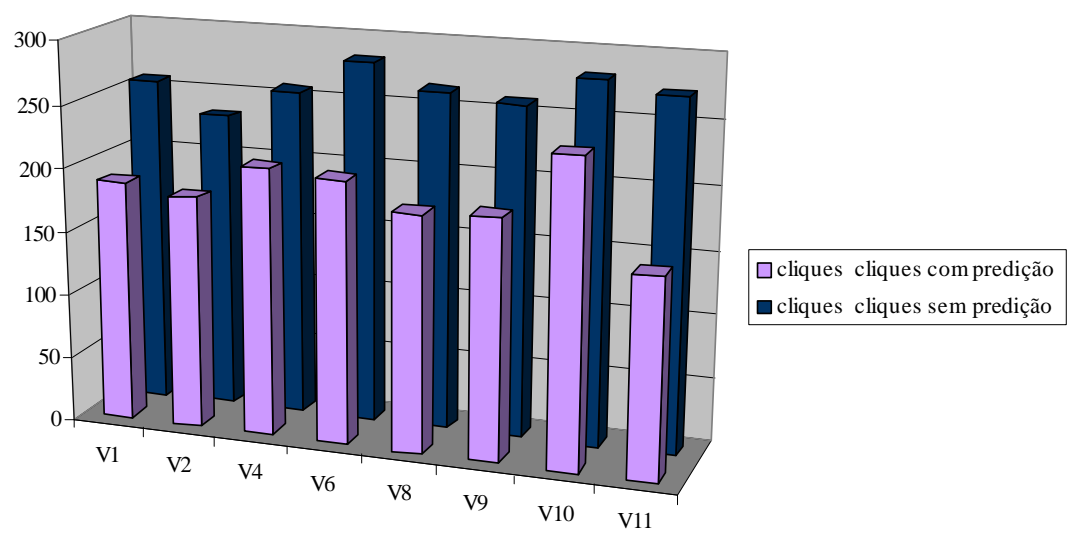

Figura 3 - Grupo 1: relação dos cliques do texto com e sem predição.

Os conjuntos de dados passíveis de análise referentes aos resultados obtidos com os voluntários do segundo grupo da escola e ensino fundamental geraram os gráficos das Figuras 4 e 5 . A Figura 4 ilustra um gráfico onde o eixo vertical refere-se ao parâmetro tempo, enquanto que na Figura 5, tal eixo faz referência ao número de cliques.

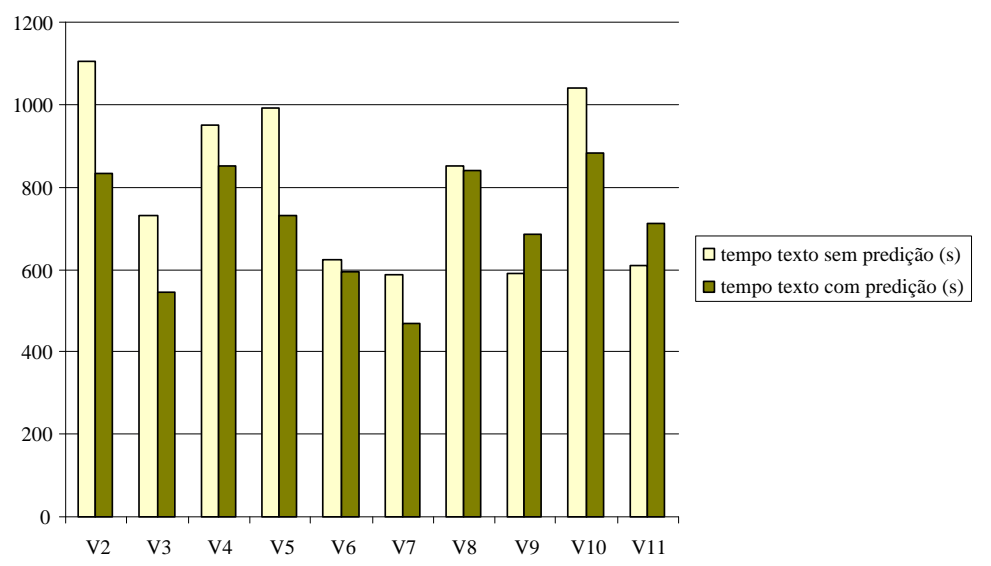

Figura 4 - Grupo 2: comparação do tempo gasto para digitação do texto com e sem predição. 
Considerando-se os valores referentes redução do número de cliques necessários para a digitação do texto do protocolo de testes, plotou-se o gráfico da Figura 5.

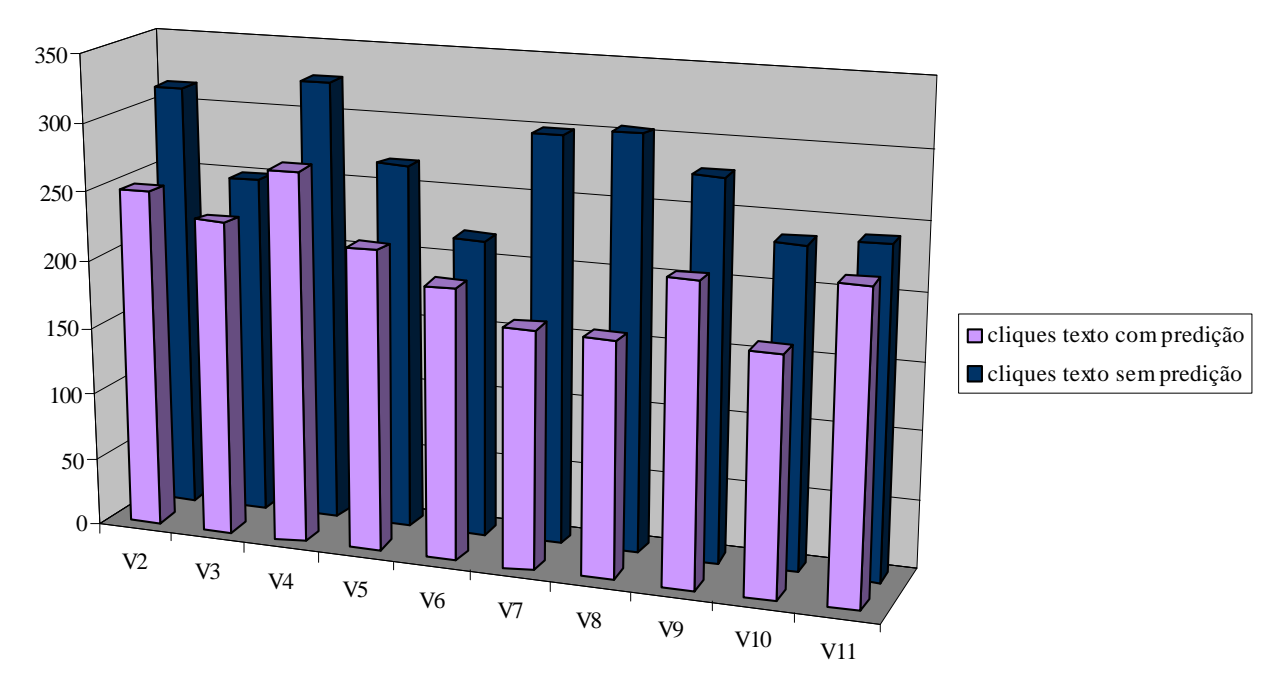

Figura 5 - Grupo 2: relação dos cliques do texto com e sem predição.

Com os resultados apresentados pel as crianças voluntárias do tercei ro grupo do ensino fundamental, para o parâmetro tempo, apontado no eixo vertical, gerou-se o gráfico da Figura 6.

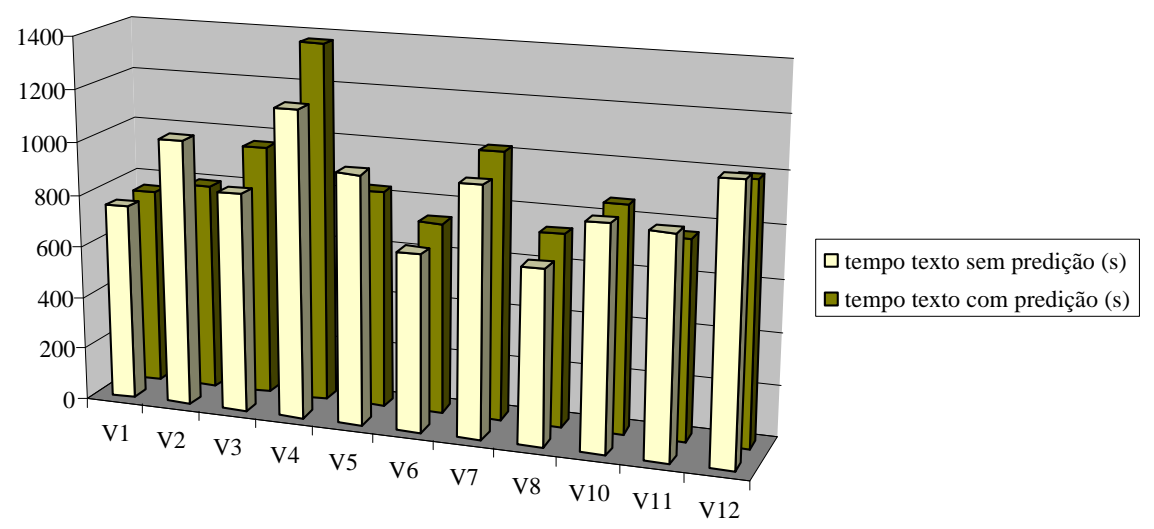

Figura 6 - Grupo 3: comparação do tempo gasto para digitação do texto com e sem predição. 
Já o parâmetro número de cliques apresentou as características ilustradas pelo eixo vertical da Figura 7.

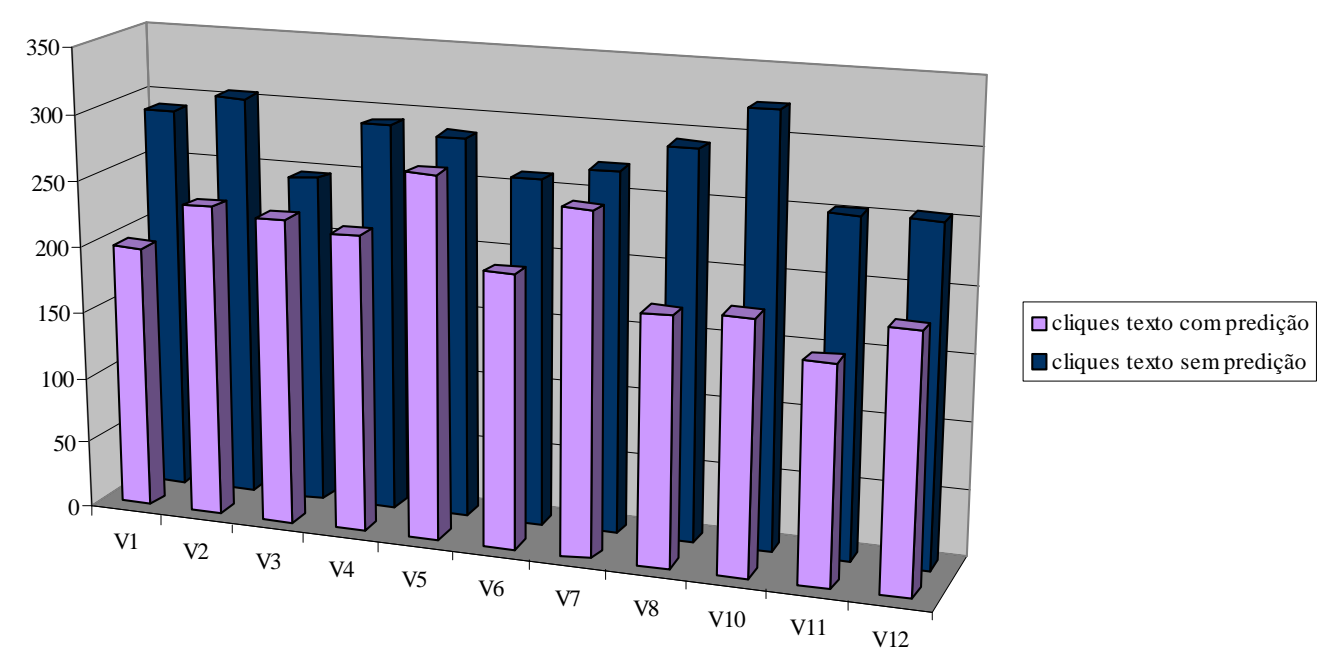

Figura 7 - Grupo 3: relação dos cliques do texto com e sem predição.

As Figuras 8 e 9 mostram os resultados obtidos pelos alunos da escola de ensino especial. Destaca-se que os Voluntários 1, 3 e 5 necessitaram que o texto fosse dividido em duas partes para a sua completa digitação sem a predição, devido ao comprometimento motor quefaz com que a digitação torne-secansativa e ansiedade apresentados na execução da tarefa; o que não ocorreu para a digitação empregando a técnica de predição.

A Figura 8 ilustra os dados considerando-se somente o parâmetro tempo, ilustrado no eixo vertical, enquanto a Figura 9 trata dos dados obtidos considerando-se o número de cliques realizado por cada voluntário. Esse parâmetro é observado no eixo vertical deste gráfico. 
JORDAN, M.; NOHAMA, P.; BRITO JÚNIOR, A.S.

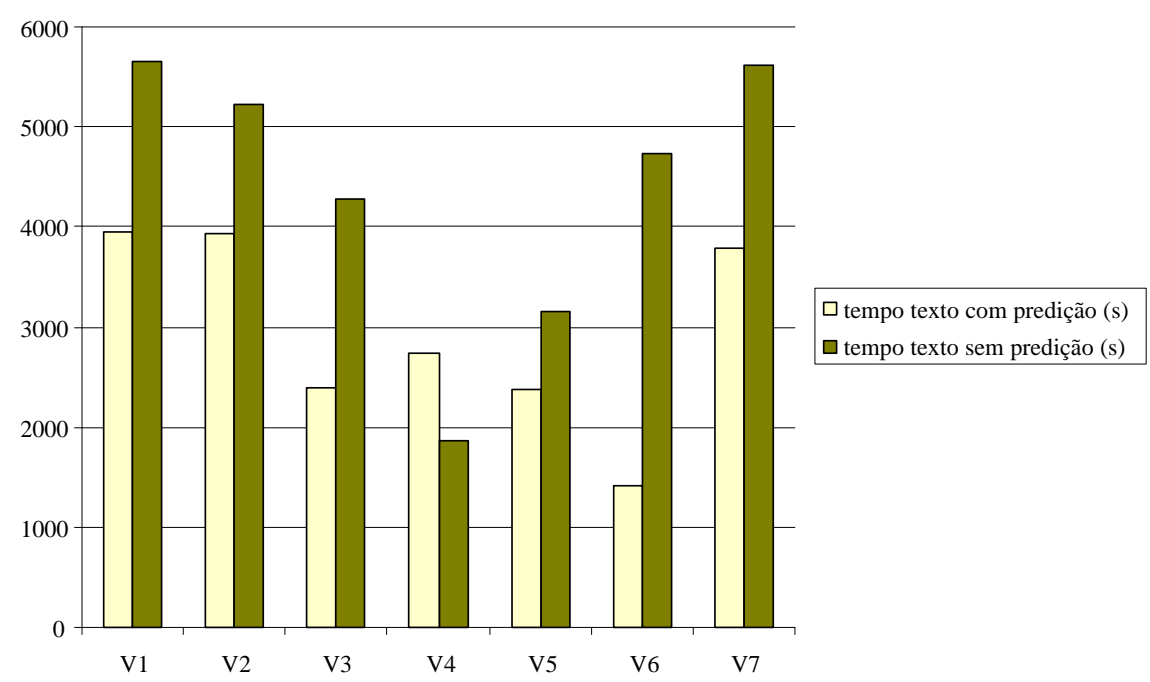

Figura 8 - Grupo 4: comparação do tempo gasto para digitação do texto com e sem predição.

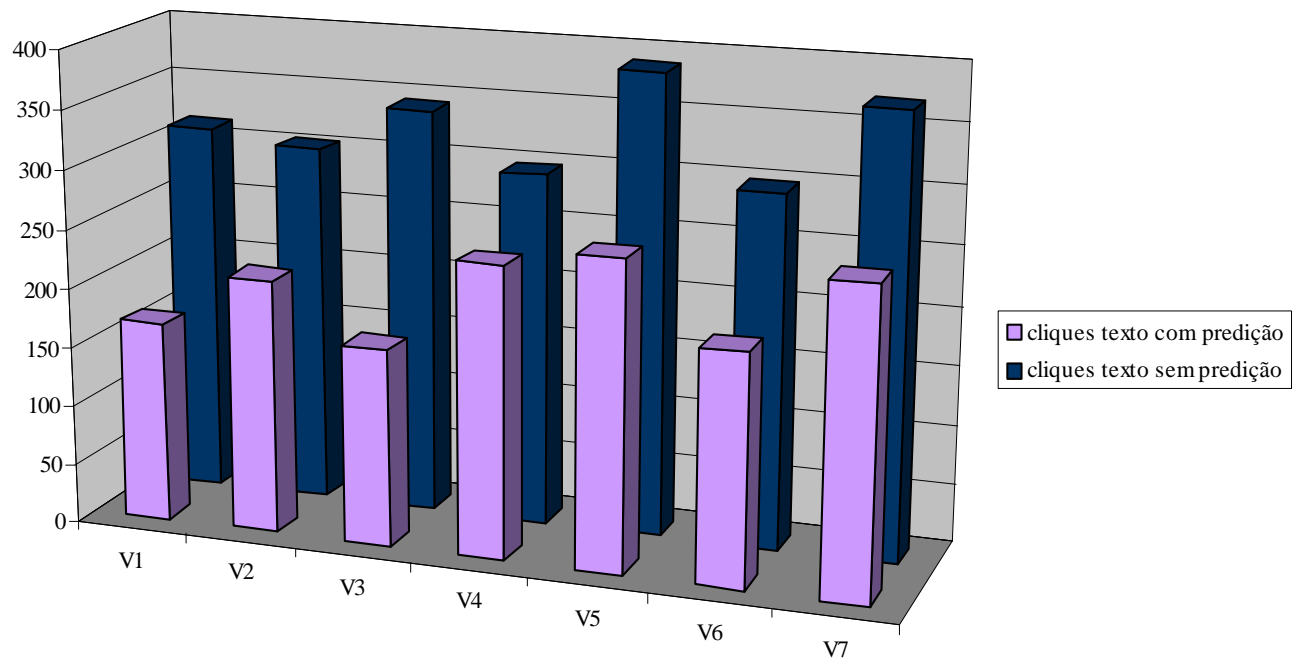

Figura 9 - Grupo 4: relação dos cliques do texto com e sem predição. 


\section{Dıscussão}

O uso de um simulador de teclado diminui o número de periféricos necessários para a produção textual e concentra em somente um campo de visão, o monitor, a atenção do usuário. Desta forma o aplicativo torna-se permitea inclusão de um número maior de usuários, além de viabilizar estudos futuros de redução de lesões por esforços repetitivos, muito comuns a usuários de computadores e amplamente citado em literatura (RIBEIRO, 1997). Assim, dispensou-se qualquer comparação entre o uso deste simulador e o teclado convencional.

Os testes deaplicabilidade da técnica de predição baseada em M odelos Ocultos de Markov apresentaram resultados positivos, tanto na redução do número de cliques quanto na aceitação do aplicativo pelos voluntários.

Do primeiro grupo da escola de ensino fundamental, somente onze crianças participaram dos testes, por atenderem aos critérios de inclusão estabelecidos edescritos em M étodo. Dos onzevoluntários, somente oito conjuntos de dados puderam ser aproveitados. Como se observa para este grupo, os resultados obtidos pelos Voluntários 3, 5 e 7 apresentaram problemas, sendo que - Voluntário 3 clicou na tecla esc do teclado convencional, fechando o aplicativo quando já estava finalizando a primeira etapa do teste, perdendo o que já havia digitado e desestimulando-o a prosseguir. A pedagoga responsável pela turma relatou ser tal criança hiperativa e apresentar problemas comportamentais em todas as aulas de informática. O Voluntário 5 não conseguiu realizar a segunda etapa do teste, por demorar na primeira etapa. Já o Voluntário 7 precisou ausentarse da sala de aula quando acabara de digitar a 15a palavra do texto.

$\mathrm{Na}$ Figura 2, observa-se que quatro voluntários necessitaram de mais tempo para preparar o texto utilizando a predição, enquanto os quatro restantes apresentaram redução neste parâmetro. A maior discrepância entre valores ocorreu com o Voluntário 11, o qual reduziu em 35,3\% o tempo de digitação com o uso da predição de palavras, e o maior acréscimo de tempo foi apresentado pelo Voluntário 2, sendo $14,4 \%$ mai or seu tempo de digitação com a predição. A redução média de tempo deste grupo foi de $17,8 \%$ enquanto o acréscimo apresentou valor médio igual a 8,2\%.

A Figura 3 ilustra a redução do número de cliques apresentada pelos oito voluntários na digitação do texto com a predição de palavras. O val or médio desta redução é de $26,3 \%$, sendo a maior economia de trabal ho apresentada pelo Voluntário 11 , com 42,9\% menos cliques.

O segundo grupo do ensino fundamental constituiu-se de 11 crianças as quais atenderam aos critérios de inclusão deste trabal ho, sendo que somente 0 conjunto de dados do Voluntário 1 não pode ser considerado. Este aluno foi descrito pela pedagoga responsável como sendo uma criança com problemas comportamentais e de aprendizado. O aluno declarou-se cansado enão conseguiu finalizar a segunda etapa do teste. 
A Figura 4 ilustra o gráfico comparativo do parâmetro tempo, no qual se observa que $80 \%$ dos voluntários apresentaram redução no tempo gasto para a digitação do texto em questão, sendo $26,4 \%$ a maior redução e $16,4 \%$ o maior acréscimo de tempo deste grupo de voluntários. $\mathrm{O}$ valor médio de redução foi de $16 \%$.

No gráfico da Figura 5, constata-se a redução do número de cliques por todos os voluntários. O valor médio desta redução é de $21 \%$, sendo o maior valor individual apresentado pelo Voluntário 8, igual a 43,3\%.

Do terceiro e último grupo da escola de ensino fundamental, 12 crianças realizaram os testes, sendo que um dos conjuntos de dados (Voluntário 9), precisou ser desconsiderado. Tal criança apresenta problemas de ansiedade, sendo acompanhada pela psicóloga da escola.

Para o parâmetro tempo elaborou-se o gráfico da Figura 6, no qual se observa que quatro voluntários apresentaram redução de tempo, um despendeu o mesmo período (em segundos) para digitar o texto com e sem a predição e os seis restantes tiveram acréscimo no parâmetro. O acréscimo médio foi de $9,7 \%$ e a redução média $12,3 \%$, sendo os máximos valores iguais a $16,8 \%$ e $22,2 \%$, respectivamente.

Na Figura 7 observa-se que, a exemplo dos outros dois grupos, todos os voluntários reduziram o número de cliques, sendo a máxima redução igual a 41,4\% (Voluntário 10), a mínima 4,9\% (Voluntário 5) e a redução média igual a $23,2 \%$.

Por motivos como ausência nas sessões de testes e ansiedade, três dos dez voluntários não finalizaram as etapas propostas, sendo formado um grupo com sete conjuntos de resultados.

Os resultad os expostos no gráfico da Figura 8 permitem a visualização da notável diferença nos valores do parâmetro tempo, sendo a maior diferença entre o tempo despendido para a digitação do texto sem e com a predição igual a 55min13s, para o Voluntário 6, e ocorrendo um acréscimo de tempo de 14min40s (Voluntário 4). Como os comprometimentos motores e cogni tivos dos voluntários são também muito diferentes e não era objetivo deste trabalho estudá-las, tal discrepância do fator tempo é considerado problema para a pesquisa. Observase que $86 \%$ dos voluntários reduziram o tempo para digitação do texto, sendo a média desta redução igual a 78\%. Somente um voluntário necessitou de maior período para digitar o texto com o uso da predição.

Na Figura 9, observa-se que o parâmetro número de cliques para a digitação do texto pelos alunos da Escola Tia Vivian Marçal sofreu pouca variação, sendo que todos os voluntários reduziram o número de cliques e a média deste parâmetro apresentou, aproximadamente, o valor de 35\%. Destaca-se que para a digitação do texto sem a predição de palavras foi necessária a divisão do texto em duas partes para três voluntários, o que não foi necessário para a digitação 
com a predição, evidenciando a diminuição da dificuldade para desempenhar esta tarefa quando comparada àquela.

Comparativamente à literatura correspondente, os valores obtidos na redução de cliques em todos os testes realizados superaram os obtidos por Fonseca e Carvalho (2002). Seus autores relatam redução média de $17 \%$ e valor máximo $34,1 \%$, contra a média de $23,5 \%$ e máximo de $43,3 \%$ do grupo de voluntários da escola de ensino fundamental e média de $34,6 \%$ e máxima de $51,3 \%$ do grupo da escola de ensino especial. Ainda em comparação ao trabal ho de Fonseca e Carvalho, este parâmetro apresentou progresso, sendo que tais pesquisadores alcançaram, com seu teclado dinâmico com predição de palavras, redução média de 10,4\%, considerando-se duas sessões de testes para treinamento do aplicativo. Ainda se deve considerar que o trabalho de Fonseca e Carvalho não tem agregada a análise gramatical, pois baseia-se no histórico inicialmente composto por uma lista de palavras a qual é incrementada no decorrer do uso do aplicativo. Destaca-se que esta limitação inicial não oferece ao usuário opções de novas palavras, não despertando sua curiosidade e criatividade. Outro problema da técnica utilizada por Fonseca e Carvalho (2002) é a possibilidade de ocorrências de erros gramaticais (como concordância verbal e nominal) e de ortografias, por não considerarem as classes gramaticais das palavras e por aceitar e adicionar à sua lista de palavras base qualquer seqüência de caracteres que o usuário digite.

\section{CONCLUSÕES}

Pessoas ativas socialmente são pessoas mais saudáveis, com elevada auto-estima e, consequentemente, de ambiente familiar mais agradável. Além disso, pessoas mais saudáveis oneram menos os cofres públicos epodem participar de ambientes antes dificultosos, como escolas e mercado de trabal ho. Com base nesses fatos, técnicas vêm sendo desenvolvidas a fim deampliar a inclusão social. Simuladores de teclado com facilitação para a produção textual permitem que deficientes verbais possam se comunicar quando o aplicativo é adicionado a um sintetizador de voz. A ampliação na produção de textos pode inserir no mercado de trabalho pessoas sem ou com limitações nos membros superiores, se tais simuladores forem associados a apontadores alternativos, como os de cabeça. Enfim, uma técnica de predição associada a um simulador de teclado pode ter aplicações diferentes para usuários diferentes, não sendo uma aplicação mais importante do que a outra.

Pelas atividades práticas que o computador oferece aos alunos, o processo ensino-aprendizagem é uma das áreas mais beneficiadas com a informática na sala de aula enos lares. Há muito tempo, Confúcio já argumentava queo aprendiz ouvee esquece, vêelembra-se; no entanto, só compreendequando faz (ISOTANI; TSUTSUMI; BRANDÃO, 2004). 
Os resultados obtidos indicam que maior quantidade e qualidade de textos também são obtidas, pois toda a predição considera regras gramaticais e o seu desenvolvimento baseia-se em um corpus criteriosamente estruturado. A qualidade do corpus esua especialidade, resultantes do processamento do mesmo, contribuem para o desenvolvimento de estudos lingüísticos. A elaboração da lista de pares de palavras versus classe gramatical do corpus estruturou a base de dados para a determinação dos parâmetros markovianos. A disponibilização de todo o desenvolvimento da técnica de predição em sítio na Internet faz com que o aplicativo seja de acesso irrestrito aos seus usuários. A limitação lingüística apontada pela utilização de um corpus pode ser ultrapassada pela abertura do código fonte, totalmente comentado, disponibilizado na Internet sob regime Livre e Gratuito (www.ler.pucpr.br/ amplisoft). Se for do desejo de algum usuário alterar a base de textos, basta ter um novo corpus, tratá-lo com as rotinas desenvolvidas neste projeto e atualizar o código fonte da técnica de predição disponível.

O trabalho realizado proporciona uma maior facilidade na produção de textos, gerando comunicação e permitindo que textos maiores possam ser preparados sem maiores dificuldades aos usuários e sem maiores esforços computacionais caracterizados por lentidão no processamento. A apreciação dos resultados permite observar o ganho de produção textual em $22 \%$ dos voluntários deficientes, os quais conseguiram reproduzir o texto solicitado em um dia ao utilizar a predição de textos, o que não ocorreu sem a predição, quando a tarefa necessitou ser dividida em dois dias para não fadigar o voluntário.

Mesmo considerando queao digitar o texto pela segunda vez o usuário já tenha conhecimento do mesmo, nota-se que crianças com diferentes condições cognitivas obtiveram melhora no desempenho ao usar a predição. Como os autores do trabalho não pertencem à área de educação, maiores detal hes não foram apurados. Pretende-se com o auxílio de profissionais da área dar continuidade à pesquisa, analisando temas do campo do ensino especial.

Os voluntários envolvidos nos testes do aplicativo demonstraram satisfação ao utilizá-lo. Essa aceitação pode facilitar a interação homem-máquina eauxiliar na comunicação escrita de pessoas com dificuldades. A análise estatística dos resultados permite visualizar a redução da quantidade de cliques, a qual indica que a quantidade de movimentos envolvidos na digitação foi reduzida, logo se reduz o esforço físico envolvido. E, ainda, indica que ocorreu o uso das palavras pertencentes à lista de predição, as quais consideram regras gramaticais; conclui-se, então, que o texto produzido apresenta qualidade gramatical.

O incremento do tempo de digitação para alguns usuários já era esperado em função da curiosidade do usuário em conhecer as palavras que aparecem na lista de predição ou pela procura da palavra na referida lista. Esta ocorrência fora descrita por Jordan, Matias e N ohama, 2006, nos testes do Teclado Virtual Livre do projeto AMPLISOFT. 
Com base na importância das inclusões digital e social acrescida dos resultados positivos obtidos nesta pesquisa, aponta-se a importância de agregar aos sistemas de comunicação um acelerador de uso eficiente, permitindo maior autonomia às pessoas consideradas não capazes por não se expressarem, seja de forma oral ou escrita, ou fazerem-no de forma lenta e dependente de outra pessoa.

\section{AgRAdecimentos}

Os autores agradecem à FINEP, ao CNPq e à SETI-PR pelo apoio financeiro e bolsas, e aos voluntários pela participação nos protocolos de testes.

\section{REFERÊNCIAS}

ALM , N.; NEWELL, A.F.; ARNOTT, J.L. Lessonsfrom applying conversation modelling to augmentative and alternative communication. In:Technology and persons with disabilities Conference, 1997, California, EUA . A nais eletrônicos. California, EUA : CSUN, 1997. Disponível em: http:/ / www.csun.edu/ cod/ conf/ 1997/ proceedings/ csun97_003.htm. A cesso emjan. 2007.

CAPOVILLA ,F.C. Pesquisa e desenvolvimento denovos recursos tecnológicos para educação especial: boas novas para pesquisadores, clínicos, professores, pais ealunos. In: Alencar, E. (Org.). Tendências e desafios de educação especial. Brasília: Secretaria deEducação Especial, 1994. p. 196-211.

FONSECA, P.M.;CARVALHO, T. Tedlado virtual dinâmico com predição. In: VII Workshop de Engenharia Biomédica, 2002, Lisboa, Portugal. A nais el etrônicos. Lisboa, Portugal, 2002. Disponível em: http:/ / www.laseeb.org/ workshops/ Biomed02/ Index.htm. A cesso emjan. 2007.

GAVA, M.L. AAC - Augmentative and alternative communication - como resposta às deficiências verbais. In: Tupy, T.M., Pravettoni, D.G. ...esefalta a pal avra, qual comunicação, qual linguagem? São Paulo: Memnon, 1999. p. 81-164.

IBGE. Censo Demográfico (2000): Características gerais da população. http:/ / www.ibge.gov.br/ home/ estatistica/ populacao/ censo2000/ default_populacao.shtm. Acesso em jan. 2007.

ISOTANI, S.; TSUTSUMI, M.; BRANDÃO, L.O. O uso do computador no ensino de geometria para deficientes auditivos. A nais do III Fórum de Informática A plicada a Pessoas Portadoras de N ecessidades Especiais, Itajaí, v.1, p. 637-642, 2004.

JORDAN , M.; MATIAS, D.H.; NOHAMA , P. A mplisoft: comunicação alternativa para todos. A nais do IV Congresso I beroamericano sobre Tecnol ogias deA poio a P ortadores de D eficiência, Vitória, v.1, 2006.

LUCCHINI, F. L. P. Controle do computador usan do movimentos do corpo identificados por um adesivo, capturados por uma câmera de vídeo W ebCam, 2001, 90f., Dissertação (Mestrado em Engenharia Elétrica e Computação) - Universi dade de Campinas - UNICAMP, Campinas, 2001. 
MORETTI, G. Princípios e significados da comunicação alternativa. In: TUPY, T.M., PRA VETTONI, D.G. ...e se falta a palavra, qual comunicação, qual linguagem? São Paulo: Memnon, 1999. p. 7-23.

NERI, M.C.; SOA RES, W.L. Idade, incapacidade e o número de pessoas com deficiência. Revista Brasileira deE studos de População, Campinas, v.21, n.2, p. 303-321, 2004.

PEÑ A-CASANOVA, J.; PAMIES, M.P. Reabilitação da afasia etranstornos associados. 2. ed. São Paulo: Manole, 2005.

RABINER, L.R. A Tutorial on hidden markov models and selected applications in speech recognition. Proceedings of the IEEE, v.77, n.2, p.257-286, 1989.

RIBEIRO, H.P. Lesões por esforços repetitivos (LER): uma doença emblemática. Cadernos de SaúdePública, Rio deJaneiro, v.13, n.2, 1997.

SARDINHA, T.B. Lingüística de corpus. São Paulo: Manole, 2004.

SCHLÜNZEN, E. T. M.; CUNHA, M.T.A.; D'OLIVEIRA, M.P.; OLIVEIRA, R.D. O Desenvolvimento de projetos e o uso do computador no ambiente de aprendizagem para crianças com necessidades especiais físicas. In: $V$ Congresso I beroamericano de Informática Educativa, 2000, Viña Del Mar, Chile. Anais eletrônicos. Viña Del Mar, Chile, 2000. Disponível em: http:/ / www.c5.cl/ ieinvestiga/ actas/ ribie2000/ papers/ 350/ index.htm. A cesso em jan. 2007.

STELLA, G. Deficiências cognitivas e uso dos procedimentos informatizados. In: TUPY, T.M., PRAVETTONI, D.G. ...e se falta a palavra, qual comunicação, qual linguagem? São Paulo: Memnon, 1999. p. 39-60. 